\title{
Broadband Wide-View LCDs With Small Color Shift
}

\author{
Meizi Jiao, Zhibing Ge, and Shin-Tson Wu, Fellow, IEEE
}

\begin{abstract}
We proposed a broadband wide-view liquid crystal display (LCD) with two biaxial films. Based on different dispersion properties of these two films, we optimized their in-plane phase retardation values to minimize the light leakage and color shift over the whole viewing cone. The broadband wide view and low color shift properties will further lead to high contrast ratio and good color quality, especially for low gray level images.
\end{abstract}

Index Terms-Biaxial film, color shift, liquid crystal display (LCD), wide view.

\section{INTRODUCTION}

W IDE view and small color shift are very important requirements for high-end liquid crystal displays (LCDs). In-plane switching (IPS) [1] and fringe-field switching (FFS) [2], [3] exhibit a comparable viewing angle, but much weaker color shift on the bright-state images than the multi-domain vertical alignment (MVA) [4] LCDs. However, their color shift for dark images remains a challenge. Different approaches using uniaxial plates [5], [6] or biaxial films [7], [8] have been proposed to enlarge viewing angles. The 100:1 isocontrast contour at an optimized wavelength (usually $\lambda \sim 550 \mathrm{~nm}$ ) can cover the entire viewing cone, however, the light leakage at other wavelengths, say red and blue, are still quite severe. As a result, the contrast ratio and color saturation are significantly degraded under white light illumination. Several approaches are proposed to reduce the dark state light leakage of a broadband light source [9], [10], but the color shift remains problematic for dark images.

In this paper, we propose a new compensation configuration for IPS/FFS LCDs using two biaxial films to reduce the dark state light leakage and color shift at off-axis viewing angles. The two films have different dispersion properties so that they can compensate each other to achieve desired effective phase retardation at all the visible wavelengths. To demonstrate the operation principles, we use the dispersion curves of polyvinyl alcohol (PVA) and polysulfone (PS) as examples, and the retardation values are optimized with the help of Poincaré sphere [11]. With our compensation scheme, both light leakage and color shift at off-axis viewing angles are reduced significantly. We also compare our results with three prior approaches: the one without any compensation film, the one with one biaxial film,

Manuscript received April 25, 2009; revised May 28, 2009. Current version published July 29, 2009. This work was supported by Chi-Mei Optoelectronics Corporation (Taiwan).

The authors are with the College of Optics and Photonics, University of Central Florida, Orlando, FL 32816, USA (e-mail: mjiao@mail.ucf.edu, zge @ creol. ucf.edu, swu@mail.ucf.edu).

Color versions of one or more of the figures in this paper are available online at http://ieeexplore.ieee.org.

Digital Object Identifier 10.1109/JDT.2009.2025608

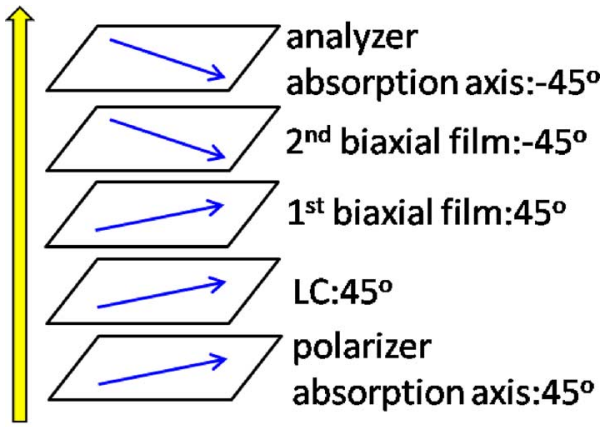

(a)

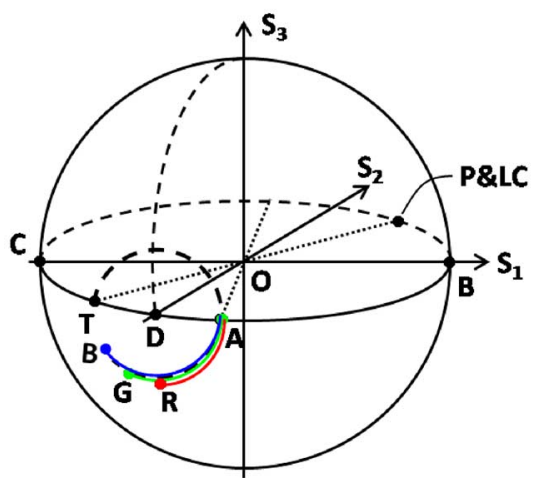

(b)

Fig. 1. (a) Compensation configuration with two biaxial films. (b) Poincare sphere representation of the broadband wide view compensation principle.

and the one with two biaxial plates [9], and find that our device configuration shows evident advantages, especially in color shift.

\section{Configuration}

Fig. 1 shows (a) the compensation configuration and (b) the compensation principles represented by Poincaré sphere. The LC cell works under a set of crossed linear polarizer system. Fig. 1(a) shows the absorption axes of the polarizer and analyzer at $45^{\circ}$ and $-45^{\circ}$, respectively. The optic axis of LC layer is parallel to the absorption axis of the bottom polarizer so that it will not change the polarization state after the polarizer no matter for normal or oblique viewing angles. The two biaxial films are parallel and perpendicular to the absorption axis of the polarizer so that the normal viewing angle image will not be disturbed by the compensation films. The $\mathrm{N}_{\mathrm{z}}$ factor, which is defined as $\left(\mathrm{n}_{\mathrm{z}}-\mathrm{n}_{\mathrm{y}}\right) /\left(\mathrm{n}_{\mathrm{x}}-\mathrm{n}_{\mathrm{y}}\right)$, is 0.5 for both films. The phase retardation difference between the first and the second biaxial films should be approximately a half-wave plate.

Under the above-mentioned conditions, the compensation principles are illustrated with Poincaré sphere in Fig. 1(b). The maximum light leakage of a crossed linear polarizer system with a LC cell occurs when viewed from the bisector direction. If the light leakage at all bisectors can be suppressed, then the 
viewing angle of the LCD can be significantly enhanced. Therefore, we optimized the compensation films by suppressing the light leakage at $\theta=70^{\circ}$ and $\varphi=270^{\circ}$, where $\theta$ is polar angle and $\lambda$ is azimuthal angle. At this angle, the transmission axis of polarizer and absorption axis of analyzer are deviated from each other, represented by point $\mathrm{T}$ and point $\mathrm{A}$ on the Poincaré sphere. Point P\&LC represent the optic axis of the polarizer and the LC layer. It is located on the extension line of OT, so the polarization state stays at point $\mathrm{T}$ after $\mathrm{LC}$ layer. Let us take $\lambda=550 \mathrm{~nm}$ green light as an example, on Poincaré sphere, the polarization state is rotated from point $T$ to point $G$ after the first biaxial film and then moved back to point $\mathrm{A}$ by the second biaxial film. As the $\mathrm{N}_{\mathrm{z}}$ factors of both films are 0.5 , the rotation axes are the same which is OD. The rotation directions however are opposite to each other due to different orientations of their optic axes. In this way, these two biaxial films can together change the polarization state from point $\mathrm{T}$ to point $\mathrm{A}$, which is the absorption axis of analyzer. Therefore the light leakage is minimized at this viewing angle. Here we used two biaxial films in order to get a wide view for other wavelengths as well. For instance, for the blue light $\lambda=450 \mathrm{~nm}$, the phase retardation values of both films are larger than that of green light for two reasons: 1) the shorter wavelength, and 2) the normal dispersion properties of the films. The second biaxial film has a larger dispersion than the first one. By adjusting the phase retardations of these two films, we can find a solution ensuring that the combination of these two films also works for blue light to change the polarization state from point $\mathrm{T}$ to point $A$, with a rotation path from point $\mathrm{T}$ to point $\mathrm{B}$ and then to point $\mathrm{A}$ on Poincare sphere. Repeating the same procedure for red light $(\lambda=650 \mathrm{~nm})$ and balancing the performance for all wavelengths, we are able to suppress the light leakage at oblique angles for the entire visible spectrum.

\section{ReSULTS}

In our calculation, the LC distribution is calculated by the finite element method and the optical properties are calculated based on extended $2 \times 2$ Jones matrix methods for both uniaxial and biaxial films [12]-[14]. The LC is homogenously aligned with cell gap $\mathrm{d}=4 \mu \mathrm{m}$. The LC parameters are listed as follows: $\mathrm{K}_{11}=9.6 \mathrm{pN}, \mathrm{K}_{22}=5.3 \mathrm{pN}, \mathrm{K}_{33}=11.6 \mathrm{pN}$, birefringence $\Delta \mathrm{n}=0.0987$ at $\lambda=550 \mathrm{~nm}$, dielectric anisotropy $\Delta \varepsilon=8.2$, and rotational viscosity $\gamma_{1}=84 \mathrm{mPa} \cdot \mathrm{s}$. We use the birefringence dispersion model [15] to include the wavelength effect

$$
\Delta n=G \frac{\lambda^{2} \lambda^{* 2}}{\lambda^{2}-\lambda^{* 2}}
$$

where $\mathrm{G}$ is a proportionality constant and $\lambda^{*}$ is the mean resonance wavelength. For the LC compounds or polymer films consisting of a single phenyl ring, their $\lambda^{*}$ is around $210 \mathrm{~nm}$.

For the biaxial films considered here, we assume the mean resonance wavelength $\lambda^{*}$ is the same for both in-plane $\left(n_{x}-n_{y}\right)$ and out-of-plane birefringence $\left(\mathrm{n}_{\mathrm{z}}-\mathrm{n}_{\mathrm{y}}\right)$. Consequently they should follow the same dispersion curve. That also explains why the $\mathrm{N}_{\mathrm{Z}}$ factor keeps almost the same over the entire visible spectrum.

We fitted the birefringence of PVA and PS based on (1), as shown in Fig. 2. The first biaxial film in our configuration has a very weak dispersion, so we chose PVA as an example, as

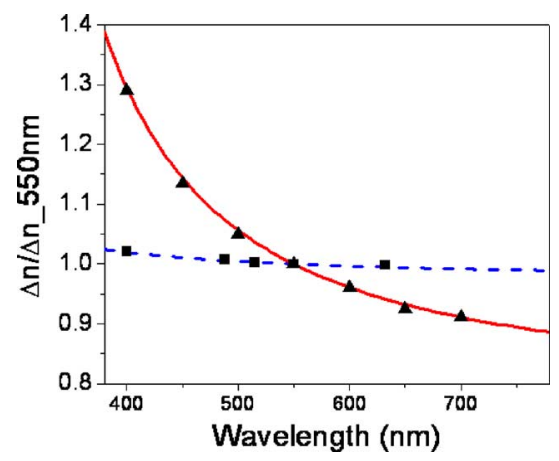

Fig. 2. Dispersion properties of the two biaxial films used in the calculations, the red solid line is the fitting curve based on data of PS (triangle dots) and the blue dashed lines are the fitting curves based on data of PVA (square dots).



Fig. 3. Maximum light leakage over the entire viewing cone of different IPS/FFS display configurations: (1) without any compensation films (black curve); (2) with one biaxial plate (blue curve); (3) with two biaxial films proposed in [9] (red curve); and (4) ours with two biaxial films (green curve).

the blue dashed lines show. While the second biaxial film has a strong dispersion and we chose polysulfone (the solid red line) as an example. The squares and triangles represent the measured data of these two materials. [16], [17] In this example, the optimized in-plane phase retardations for the two biaxial films are $740 \mathrm{~nm}$ and $460 \mathrm{~nm}$ for the first and second biaxial films at $\lambda=550 \mathrm{~nm}$.

To show the compensation performance, in Fig. 3 we plotted the maximum light leakage over the whole viewing cone within the visible spectrum. The results are compared with three prior approaches. Curve 1 represents the dark state light leakage of an IPS or FFS display without any compensation film. There is strong off-axis light leakage at any wavelength. With one biaxial film compensated, as curve 2 shows, the light leakage is significantly reduced at the optimized wavelength but still remains relatively severe at other wavelengths. This will further degrade the contrast and the color uniformity. Curve 3 shows the light leakage of compensation configuration with two biaxial films proposed in [9]. In this configuration, each biaxial film contributes to half of the phase needed. We used the dispersion curve of PVA in Fig. 2 to get an optimized result of their configuration. Curve 4 is for our compensation configuration with two biaxial films. Both configurations with two films can reduce the light leakage significantly over the entire visible spectrum. This will result in high contrast over the whole viewing cone under white light. Moreover, the broadband weak light leakage will help to reduce the color shift of low gray level images.

Dark state colors at oblique viewing angles can vary from that at normal viewing angle, depending on the ratio of the light 

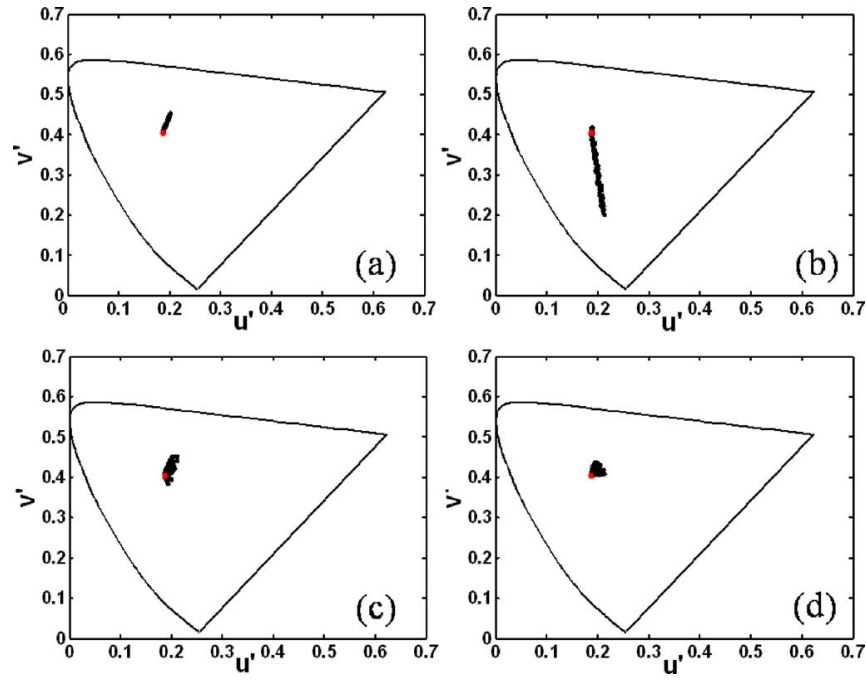

Fig. 4. Dark state color shift at the varied viewing angles from $0^{\circ}$ to $60^{\circ}$ of different display configurations: (a) without any compensation film; (b) with one biaxial plate; (c) with two biaxial films proposed in [9]; and (d) ours with two biaxial films. Red spot represent the color at normal viewing angle.

leakage at different wavelengths. Fig. 4 compares the dark state color shift of four IPS/FFS configurations. The CIE 1976 uniform chromaticity scale diagram is used here to give a direct view of the color difference. The red dots are the $\left(\mathrm{u}^{\prime}, \mathrm{v}^{\prime}\right)$ coordinates at normal viewing angle and those black dots correspond to the $\left(\mathrm{u}^{\prime}, \mathrm{v}^{\prime}\right)$ values at viewing angles with $\theta$ varied from $0^{\circ}$ to $60^{\circ}$ and $\varphi$ from $0^{\circ}$ to $360^{\circ}$. The deviation between the red dots and black dots represent the color shift between normal and oblique viewing angles, as described in

$$
\Delta u^{\prime} v^{\prime}=\sqrt{\left(u^{\prime}{ }_{2}-u_{1}^{\prime}\right)^{2}+\left(v_{2}^{\prime}-v_{1}^{\prime}\right)^{2}} .
$$

where $\left(\mathrm{u}_{1}^{\prime}, \mathrm{v}_{1}^{\prime}\right)$ is the coordinate at normal viewing angle and $\left(\mathrm{u}_{2}^{\prime}, \mathrm{v}_{2}^{\prime}\right)$ is that at an oblique viewing angle.

The maximum color shift $\Delta \mathrm{u}^{\prime} \mathrm{v}^{\prime}$ is 0.051 for the configuration without compensation film, as Fig. 4(a) shows. As in Fig. 4(b), it increases to 0.206 in the configuration with one biaxial plate, due to the strong blue shift resulting from the strong light leakage at shorter wavelengths compared with longer wavelengths. The two biaxial films configuration proposed in [9] improves the color uniformity to a level similar to crossed polarizers, with a maximum color shift $\Delta \mathrm{u}^{\prime} \mathrm{v}^{\prime}=0.052$. For comparison, the dark state color shift of our configuration is reduced significantly to $\Delta \mathrm{u}^{\prime} \mathrm{v}^{\prime}=0.033$, as shown in Fig. 4(d). With better material engineering of the two biaxial films, the color shift can be even further reduced in our configuration.

\section{CONCLUSION}

We have proposed a new compensation configuration for LCDs with homogenous alignment using two biaxial films. The proposed configuration can achieve achromatic dark state and good color uniformity at different viewing angles. The greatly suppressed light leakage over the entire visible spectrum can further help to reduce the color shift of low gray level images of IPS/FFS LCDs.

\section{REFERENCES}

[1] R. A. Soref, "Field effects in nematic liquid crystals obtained with interdigital electrodes," J. Appl. Phys., vol. 45, pp. 5466-5468, Dec. 1974.

[2] S. H. Lee, S. L. Lee, and H. Y. Kim, "Electro-optic characteristics and switching principle of a nematic liquid crystal cell controlled by fringefield switching," Appl. Phys. Lett., vol. 73, pp. 2881-2883, Nov. 1998.

[3] Z. Ge, S. T. Wu, S. S. Kim, J. W. Park, and S. H. Lee, "Thin cell fringefield-switching liquid crystal display with a chiral dopant," Appl. Phys. Lett., vol. 92, p. 181109, May 2008.

[4] R. Lu, S. T. Wu, and S. H. Lee, "Reducing the color shift of a multidomain vertical alignment liquid crystal display using dual threshold voltages," Appl. Phys. Lett., vol. 92, p. 051114, 2008.

[5] J. E. Anderson and P. J. Bos, "Methods and concerns of compensating in-plane switching liquid crystal displays," Jpn. J. Appl. Phys., vol. 39 pp. 6388-6392, Nov. 2000.

[6] X. Zhu, Z. Ge, and S. T. Wu, "Analytical solutions for uniaxial-filmcompensated wide-viewing liquid crystal displays," J. Display. Tech., vol. 2, no. 1, pp. 2-20, Mar. 2006.

[7] Y. Saitoh, S. Kimura, K. Kusafuka, and H. Shimizu, "Optimum film compensation of viewing angle of contrast in in-plane-switching-mode liquid crystal display," Jpn, J. App. Phys., vol. 37, pp. 4822-4828, Sep. 1998.

[8] D. Kajita, I. Hiyama, Y. Utsumi, K. Miyazaki, M. Hasegawa, and M. Ishii, "An IPS-LCD with a high contrast ratio of over 80:1 at all viewing angles," J. Soc. Inf. Disp., vol. 15/2, pp. 139-144, 2007.

[9] T. Ishinabe, T. Miyashita, and T. Uchida, "Wide-viewing-angle polarizer with a large wavelength range," Jpn. J. Appl. Phys., vol. 41, pp. 4553-4558, 2002.

[10] J. H. Lee, H. Choi, S. H. Lee, J. C. Kim, and G. D. Lee, "Optical configuration of a horizontal-switching liquid-crystal cell for improvement of the viewing angle," Appl. Opt., vol. 45, pp. 7279-7285, Oct. 2006.

[11] K. Vermeirsch, A. D. Meyere, J. Fornier, and H. D. Villeeschouwer, "Viewing angle of liquid-crystal displays: Representation on the Poincaré sphere," Appl. Opt., vol. 38, pp. 2775-2786, 1999.

[12] A. Lien, "Extended Jones matrix representation for the twisted nematic liquid-crystal display at oblique incidence," Appl. Phys. Lett., vol. 57, pp. 2767-2769, 1990.

[13] Z. Ge, T. X. Wu, X. Zhu, and S. T. Wu, "Reflective liquid crystal displays with asymmetric incidence and exit angles," J. Opt. Soc. Am. A., vol. 22, pp. 966-977, 2005.

[14] M. Jiao, Z. Ge, and S. T. Wu, "Wide-view MVA-LCDs with an achromatic dark state," J. Display Technol., vol. 5, no. 5, pp. 141-146, May 2009.

[15] S. T. Wu, "Birefringence dispersions of liquid crystals," Phys. Rev. A., vol. 33, pp. 1270-1274, 1986.

[16] S. T. Wu, "Phase-matched compensation films for liquid crystal displays," Mat. Chem. Phys., vol. 42, pp. 163-168, 1995.

[17] H. Muraji, H. Koh, T. Kuwata, Nakagawa, S. lhara, and Y. Kurisu, "Color VGA F-STN display with fast response time and high contrast ratio by using MLS method," SID Dig. Tech. Papers, vol. 25, pp. 61-64, 1994.

Meizi Jiao received the B.S. degree in optics from Zhejiang University (ZJU), Hangzhou, China, in 2006, and is currently working toward the Ph.D. degree at the College of Optics and Photonics, University of Central Florida, Orlando.

Her current research interests include optical compensation films for wideview LCDs, and fast-response LC devices.

Zhibing Ge (S02) received the B.S. degree in electrical engineering from Zhejiang University, Hangzhou, China, in 2002, and the M.S. and Ph.D. degrees in electrical engineering from the University of Central Florida, Orlando, in 2004 and 2007, respectively.

Since 2008, he has been with the College of Optics and Photonics at University of Central Florida as a research scientist. His research interests include novel liquid crystal displays and laser beam steering technologies. He has published 1 book chapter, over 30 journal papers, and 12 issued or pending patents in related area.

Dr. Ge is a recipient of the 2008 Otto Lehmann Award. Since May 2008, he has served as an associate editor for Journal of Society for Information Display (JSID) on the LCD division. 


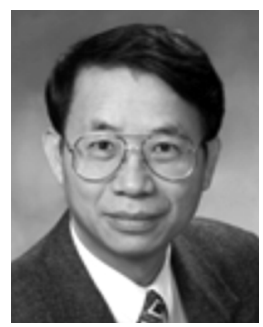

Shin-Tson Wu (M'98-SM'99-F'04) received the B.S. degree in physics from National Taiwan University, and the Ph.D. degree from the University of Southern California, Los Angeles.

$\mathrm{He}$ is a PREP professor at College of Optics and Photonics, University of Central Florida (UCF). His studies at UCF concentrate in liquid crystal displays, liquid crystal materials, optical communications, photonic crystal fibers, and bio-photonics. Prior to joining UCF in 2001, he worked at Hughes Research Laboratories, Malibu, CA, for 18 years. He has co-authored 5 books: Introduction to Flat Panel Displays (Wiley, 2008, with J. H. Lee and D. N. Liu), Fundamentals of Liquid Crystal Devices (Wiley, 2006, with D. K. Yang); Introduction to Microdisplays (Wiley, 2006, with D. Armitage and I. Underwood), Reflective Liquid Crystal Displays (Wiley, 2001, with D. K. Yang) and Optics and Nonlinear Optics of Liquid Crystals (World Scientific, 1993, with I. C. Khoo), as well as 6 book chapters, over 300 journal publications, and more than 55 issued patents.

Dr.Wu is a recipient of SPIE G. G. Stokes award and SID Jan Rajchman prize. $\mathrm{He}$ is a Fellow of the Society of Information Display (SID), Optical Society of America (OSA), and SPIE. 